\title{
Decreased Vision as Initial Presenting Symptom of Acute Lymphoblastic Leukemia: A Case Report
}

\author{
Christoph Palme Nikolaos E. Bechrakis Martin Stattin Gertrud Haas \\ Claus Zehetner
}

Department of Ophthalmology, Medical University Innsbruck, Innsbruck, Austria

\section{Keywords}

Acute lymphoblastic leukemia · Visual disorder · Optical coherence tomography · Serous macular detachment

\section{Abstract}

This case illustrates that hematologic disorders must be considered as a potentially lifethreatening cause for vision loss. Proper laboratory workup and timely interdisciplinary approach are essential to ensure the best possible care for ophthalmic patients. Historically, before the use of bone marrow biopsy, the ophthalmologist was often asked to assist in the diagnosis of leukemia. Since ophthalmological symptoms may be the initial presenting signs of leukemia as highlighted in this case, the ophthalmogist is still of crucial importance. 


\section{Case Reports in Ophthalmology}

\section{Introduction}

Potentially life-threatening systemic diseases like hematologic disorders can initially present with isolated ocular symptoms. Therefore, accurate ophthalmologic assessment and prompt ancillary testing facilitates timely diagnosis and subsequent therapy in undefined cases.

Thus, we report of a patient presenting with isolated decreased monocular vision. Ophthalmic and clinical workup led to the diagnosis of acute lymphoblastic leukemia (ALL). Only a sparse number of similar cases have been reported in the literature [1-5]. We would like to elucidate the striking correlation between the amount of subretinal fluid, total leukocyte count and visual acuity.

\section{Case Presentation}

The 28-year-old female patient presented with decreased central vision in her right eye since getting up that morning. She reported no other symptoms and stated to be in good health. Her family, social and past medical history revealed no relevant findings.

The initial corrected visual acuities were 20/40 (OD) and 20/20 (OS), but it dropped to 20/200 (OD) on day 5 of clinical follow-up.

Ophthalmic examinations of the anterior segment of both eyes showed no pathological findings. Fundus examination showed a vital optic nerve head, but the macula of the right eye presented with a prominent center-involving serous neurosensory retinal detachment and whitish-yellowish choroidal infiltrations. It was less prominent in the central macula of the left eye, but extensive serous detachment was present in the area of the papillomacular bundle (fig. 1) at the inferotemporal vascular arcade. Optical coherence tomography (OCT) confirmed prominent subretinal fluid overlying an area of choroidal thickening. Fluorescein angiography detected multifocal areas of leakage at the level of the retinal pigment epithelium (fig. 1).

Due to her initial findings, the primary care ophthalmologist considered central serous retinopathy or chorioretinitis of unknown origin as potential differential diagnoses. Followup on day 3 revealed further deterioration of symptoms despite proper treatment with oral prednisone and ketorolac eyedrops. Upon referral, immediate laboratory workup was initiated. The leukocyte count (normal range in parentheses) was found to be substantially increased to a total of $38.2 \mathrm{~g} / \mathrm{l}(4.0-10.0)$ [erythrocytes $4.65 \mathrm{~g} / \mathrm{l}(3.8-4.2)$, thrombocytes $30 \mathrm{~g} / \mathrm{l}$ $(150-380)$, blasts $87 \%(0.0)$ and increased LDH $645 \mathrm{U} / \mathrm{l}(100-250)]$. Considering this lifethreatening situation, the patient was admitted to the department of hematology. Upon further enquiry and more detailed medical history, the patient remembered an ill-defined swelling of several lymph nodes.

Ancillary clinical testing confirmed the diagnosis of common B-cell ALL. The Bcr/ABL oncogene was tested to be negative. Chemotherapy was inducted on day 5 according to the GMALL 03/07 study protocol [6]. On consecutive ophthalmic examinations, her vision dropped in both eyes as shown in figure 2. The clinical findings and OCT images demonstrated a striking relationship between visual acuity, amount of subretinal fluid and leukocyte count. To depict this significant correlation, the thickness of submacular fluid was measured and compared to vision and leukocyte count (fig. 2). 


\section{Case Reports in Ophthalmology}

Case Rep Ophthalmol 2016;7:377-383

(c) 2016 The Author(s). Published by S. Karger AG, Basel www.karger.com/cop

Palme et al:: Decreased Vision as Initial Presenting Symptom of Acute Lymphoblastic Leukemia: A Case Report

\section{Discussion}

Leukemia is a potential life-threatening malignant disease characterized by uncontrolled proliferation and accumulation of mature and premature malignant leukocytes in the bone marrow with a subsequent peripheral distribution. Different forms of leukemia can be classified by their cell of origin and by their time of duration. Detailed subclasses are described by their genetic and immunochemical characteristics $[7,8]$. The overall incidence rate of ALL is $1.28 / 100,000$ per year showing a bimodal distribution with higher rates in younger ( $0-14$ years) and older patients (over 54 years) [9].

Ocular involvement of ALL can affect all intraocular structures. Direct leukemic manifestations as infiltrates have been reported of the optic nerve, choroid, retina, iris, ciliary body and anterior chamber. Indirect leukemic manifestations such as central serous chorioretinopathy overlaying choroidal infiltrations, retinal vascular sheathing and subconjunctival, anterior chamber, intraretinal, or intravitreal hemorrhages have been found. These findings may be related to accompanying anemia, thrombocytopenia or hyperviscosity states [10].

Histopathology of postmortem specimens performed by Kincaid and Green [10] at the Wilmer Ophthalmological Institute between 1923 und 1980 found ocular involvement in $82 \%$ of patients with ALL. However, ocular examinations at the time of diagnosis show less frequent ocular manifestations. Reddy et al. [11] reported ocular findings in $35.4 \%$ of 288 newly diagnosed cases of leukemia in children and adults, but obvious clinical symptoms were only present in 10\%. Ocular involvement in hematologic disease entities may benefit from immediate systemic chemotherapy of ALL as they are usually not treated directly [10].

The leukemic infiltrates were found in almost any location in the eye; however, they are most frequent in the choroid with primary or compressive involvement of the adjacent choriocapillaris [1]. Blood dyscrasia and leukemic cell infiltration may cause focal choroidal ischemia. The consecutive dysfunction of the retinal pigment epithelium and the outer blood-retina barrier may ultimately lead to serous retinal detachment as observed in our patient $[12,13]$. These findings correlate well with the multifocal areas of leakage at the level of the retinal pigment epithelium shown by fluorescein angiography in our case. Similar pathomechanisms are discussed in central serous retinopathy or paraproteinemic maculopathy [14]. Mansour et al. [14] proposed another potential pathomechanism contributing to serous retinal detachment. They suggested that an increased osmotic gradient caused by subretinal accumulation of high-molecular-weight immunoglobulins induces exudation.

Due to immediate induction of chemotherapy by the department of oncology and hematology, the leukocyte count of the patient declined adequately. Upon her last visit to the department of ophthalmology her visual acuity improved to 20/40 (OD) and 20/20 (OS), and the reduction of subretinal fluid showed an evidently positive correlation with regressing leukocyte counts (fig. 2). In line with the therapeutic response to chemotherapy, her ocular biomicroscopic examination, OCT and fluorescein angiography had resolved back to normal (fig. 3).

\section{Statement of Ethics}

This case report was conducted in accordance with good clinical practices. The authors state to have full control of all primary data and have no ethical conflicts to disclose. 
Case Reports in
Ophthalmology

Case Rep Ophthalmol 2016;7:377-383 $10.1159 / 000447994$

(C) 2016 The Author(s). Published by S. Karger AG, Basel www.karger.com/cop

Palme et al.: Decreased Vision as Initial Presenting Symptom of Acute Lymphoblastic Leukemia: A Case Report

\section{Disclosure Statement}

The authors have declared no conflicts of interest.

\section{References}

1 Adam MK, Pitcher JD, Shields CL, Maguire JI: Enhanced depth imaging optical coherence tomography of precursor cell leukemic choroidopathy before and after chemotherapy. Middle East Afr J Ophthalmol 2015;22:249-252.

2 Smith AG, London NJS, Pao K, Lipkowitz J, Kurup SK, Garg SJ: Leukemia presenting as serous retinal detachment. Retin Cases Brief Rep 2014;8:279-282.

-3 Lee CS, Shim JW, Yoon JS, Lee SC: Acute lymphoblastic leukemia presenting as bilateral serous macular detachment and lacrimal gland enlargement. Can J Ophthalmol 2012;47:e33-e35.

-4 Golan S, Goldstein M: Acute lymphocytic leukemia relapsing as bilateral serous retinal detachment: a case report. Eye 2011;25:1375-1378.

5 Kim J, Chang W, Sagong M: Bilateral serous retinal detachment as a presenting sign of acute lymphoblastic leukemia. Korean J Ophthalmol 2010;24:245-248.

-6 Gökbuget N, Hoelzer D, Arnold R, Böhme A, Bartram CR, Freund M, et al: Treatment of Adult ALL according to protocols of the German Multicenter Study Group for Adult ALL (GMALL). Hematol Oncol Clin North Am 2000;14:1307-1325, ix.

7 Béné MC, Nebe T, Bettelheim P, Buldini B, Bumbea H, Kern W, et al: Immunophenotyping of acute leukemia and lymphoproliferative disorders: a consensus proposal of the European LeukemiaNet Work Package 10. Leukemia 2011;25:567-574.

8 Chiaretti S, Zini G, Bassan R: Diagnosis and subclassification of acute lymphoblastic leukemia. Mediterr J Hematol Infect Dis 2014;6:e2014073.

-9 Sant M, Allemani C, Tereanu C, De Angelis R, Capocaccia R, Visser O, et al: Incidence of hematologic malignancies in Europe by morphologic subtype: results of the HAEMACARE project. Blood 2010;116:3724-3734.

10 Kincaid MC, Green WR: Ocular and orbital involvement in leukemia. Surv Ophthalmol 1983;27:211232.

11 Reddy SC, Jackson N, Menon BS: Ocular involvement in leukemia - a study of 288 cases. Ophthalmologica 2003;217:441-445.

12 Rosenthal AR: Ocular manifestations of leukemia. A review. Ophthalmology 1983;90:899-905.

-13 Guymer RH, Cairns JD, O’Day J: Benign intracranial hypertension in chronic myeloid leukemia. Aust NZ J Ophthalmol 1993;21:181-185.

14 Mansour AM, Arevalo JF, Badal J, Moorthy RS, Shah GK, Zegarra H, et al: Paraproteinemic maculopathy. Ophthalmology 2014;121:1925-1932. 


\section{Case Reports in Ophthalmology}
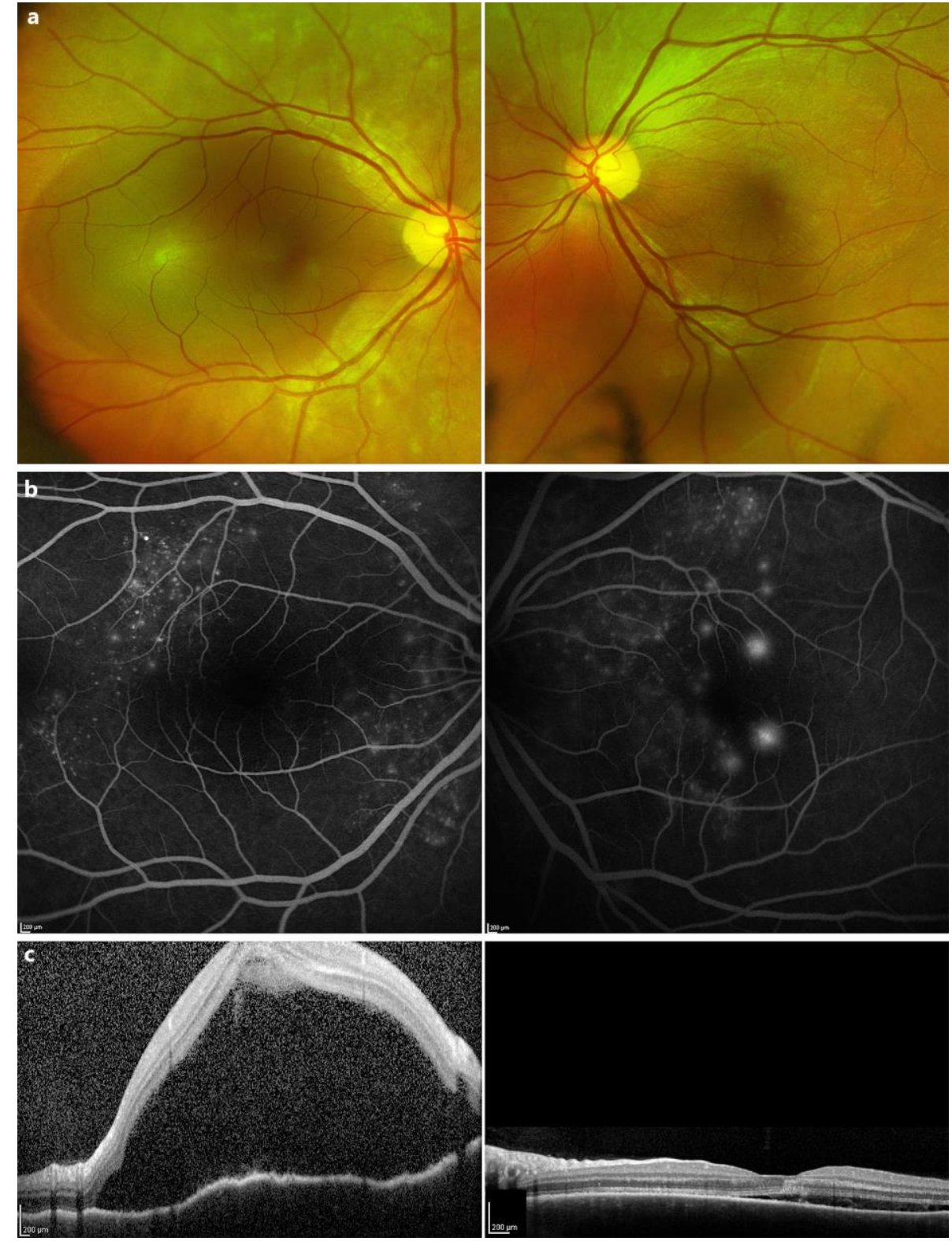

Fig. 1. a Fundus photographs of the right eye (left) and the left eye (right) showing bilateral serous retinal detachment on day 4. b Fluorescein angiography detects a 'Milky-Way pattern' of diffuse small punctate hyperfluorescence with increasing size and brightness on both eyes as the study progresses on day 1 . c OCT shows more prominent submacular fluid on the right eye compared to the left eye with maximal detection on day 4 . 
Palme et al.: Decreased Vision as Initial Presenting Symptom of Acute Lymphoblastic Leukemia: A Case Report
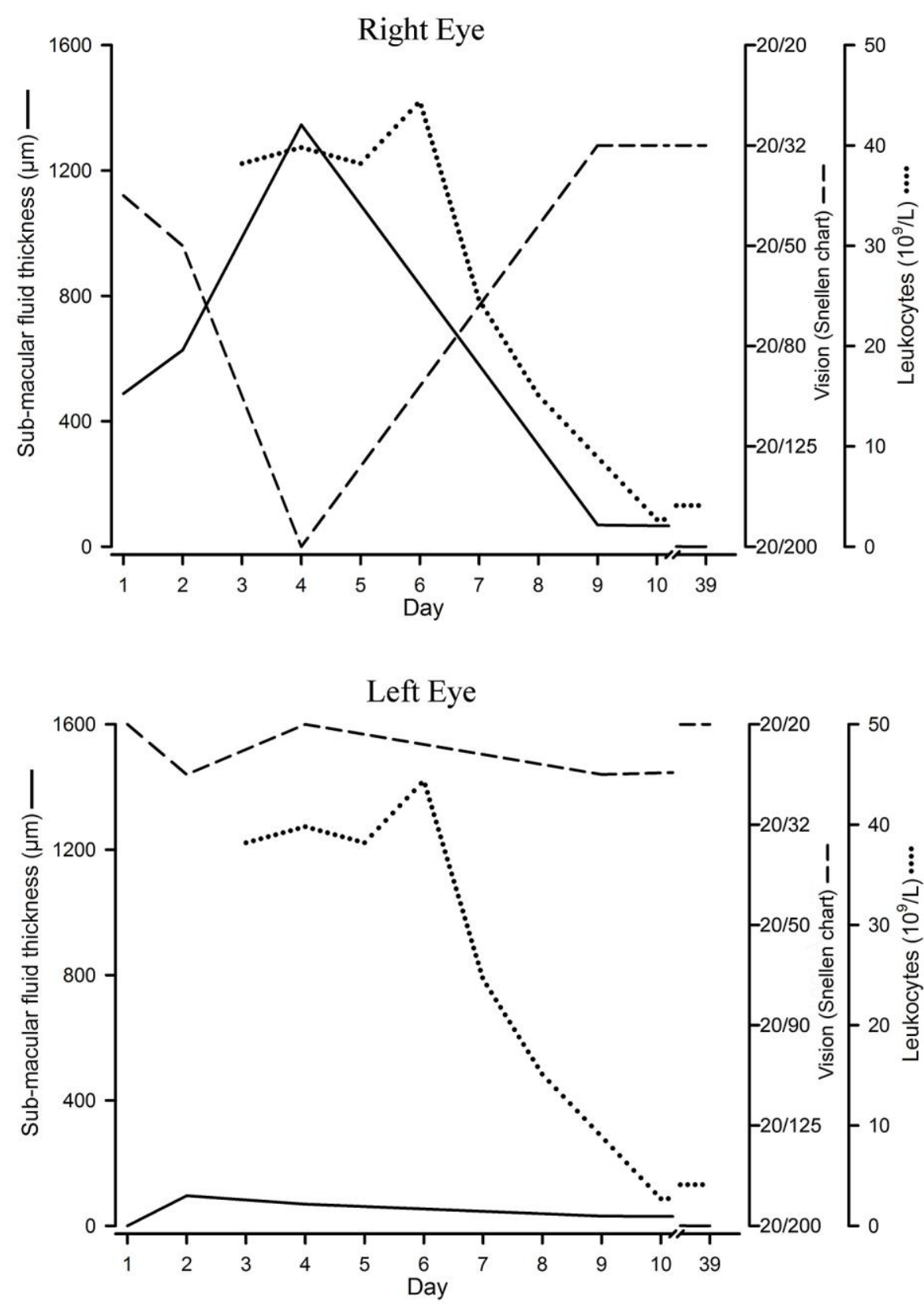

Fig. 2. These figures demonstrate the correlation of best corrected visual acuity (BCVA - measured by the Snellen chart; - -), amount of submacular fluid (----) and leukocyte count ( $\bullet . \bullet$ ) of the right eye (above) and the left eye (below). On day 3, lab work is initiated. After induction of chemotherapy, leukocyte count drastically declines, whereas BCVA significantly improves due to decreased submacular fluid. Additionally, these figures demonstrate the unilateral onset of vision deterioration. Examinations reveal a central onset of increased submacular fluid on the right eye compared to the more peripheral onset on the left eye. Hence, the effect on central vision should be worse on OD compared to OS. 


\section{Case Reports in Ophthalmology}

\begin{tabular}{l|l}
\hline Case Rep Ophthalmol 2016;7:377-383 \\
\hline $10.1159 / 000447994$ & $\begin{array}{l}\text { ○ } 2016 \text { The Author(s). Published by S. Karger AG, Basel } \\
\text { www.karger.com/cop }\end{array}$ \\
\hline
\end{tabular}

Palme et al.: Decreased Vision as Initial Presenting Symptom of Acute Lymphoblastic Leukemia: A Case Report
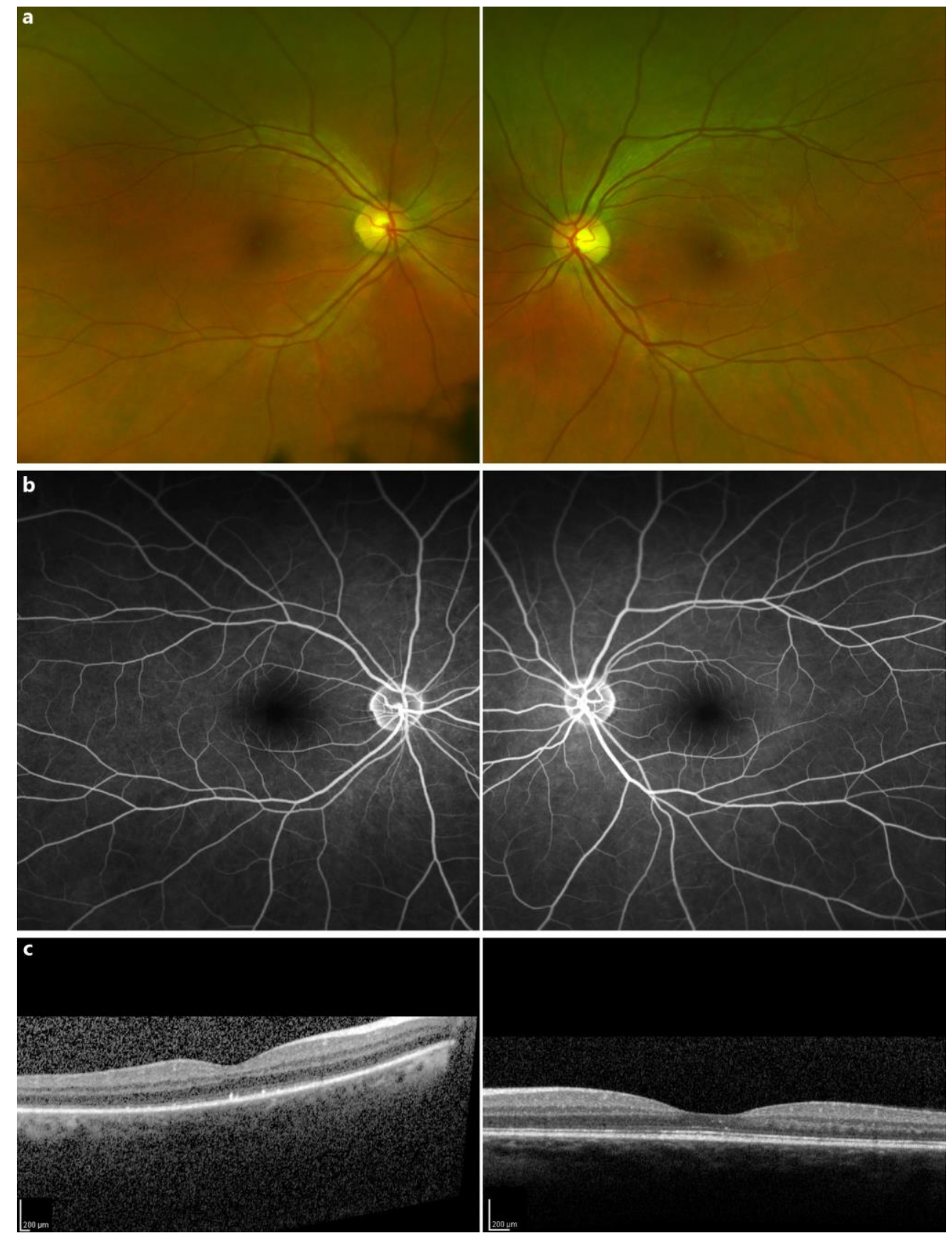

Fig. 3. a Fundus photographs of the right eye (left) and the left eye (right) together with fluorescein angiography (b) and OCT (c) have resolved back to normal on day 39 . Only small exudates are discernible after complete resorption of submacular fluid. 